\begin{tabular}{lrr} 
STUDIA & ROMANICA & POSNANIENSIA \\
\hline UAM & Vol. 29 & Pozlań 2003
\end{tabular}

ANNA LOBA

Universitẻ Adam Mickiewicz ả Poznań

\title{
LE PROJET DU BONHEUR CONJUGAL DANS LE MESNAGIER DE PARIS
}

\begin{abstract}
A b stra c t. Loba Anna, Le projet du bonheur conjugal dans «Le Mesnagier de Paris» [Project of conjugal happiness in Le Mesnagier de Paris]. Studia Romanica Posnaniensia, Adam Mickiewicz University Press, Poznań, vol. XXIX: 2003, pp. 31-40, ISBN 83-232-1232-5, ISSN 0137-2475.
\end{abstract}

Mesnagier de Paris, drafted in 1393 by an unknown bourgeois person for his young wife, is considered as the most famous medieval treaty concerning the education of women. The happiness is not the main subject of Mesnagier; it is not even evoked in a direct way. The happiness or bliss remains an objective presupposed by the advice given to the young wife. The main purpose of all the eductation is order. The happiness is its corollary. The vision of bliss sketched in Mesnagier de Paris concentrates around the image of the house. The project of the bourgeois authors thus is to build through the structure of the domestic space in its material and symbolic dimension a vision of the deserving and honest life. The conception of happiness is based on an at the same time medieval and bourgeois vision of the world. The idea of the stable and unchanging character of the relations between the sexes and that of the ambivalent nature of women - are medieval. However the ecstasy of conjugal happiness softens in a sensitive way the usual moralizing rhetoric. The need of prosperity and material comfort, declared openly, is connected to the urban culture that gets ready for the splendors of the Renaissance.

Le Mesnagier de Paris est un manuel qui réunit des enseignements domestiques et moraux, rédigés en 1393 par un bourgeois anonyme, probablement officier royal, pour son jeune épouse de quinze ans ${ }^{1}$. À côté du Livre pour l'enseignement de ses filles composé à la même époque (1371) par un noble angevin, Geoffroi de La Tour Landry, le Mesnagier est considéré comme le plus fameux traité médiéval portant sur l'éducation des femmes.

' Les historiens soulignent une grande différence d'âge entre les époux au Moyen Age: «Partout, l'élévation de l'âge au mariage des garçons est causée par la ponction qu'exercent les hommes d'âge mûr sur la génèration des adolescentes, tandis que la compétition entre les pères pour placer les filles entraîne l'abaissement de l'âge des jeunes êpouses et l'augmentation du poids des dots, partout en hausse sensible au XVIe siècle». (H. Bresc, L'Europe des villes et des campagnes (XIII $-X V^{e}$ siècle), in Histoire de la famille, sous la direction de A. Burguière, Ch. Klapisch-Zuber, M. Segalen, F. Zonabend, t. I, Colin, Paris 1986, p. 402). 
Le Mesnagier de Paris suivant le modèle d'un traité juridique ou d'une somme théologique est réparti en distinctions et subdivisé en articles. Il se compose de trois parties dont la première contenant neuf articles se réfere à la conduite et aux devoirs d'une épouse chrétienne, la seconde, avec ses cinq articles, présente les responsabilités d'une maîtresse de maison et la troisième est dédiée aux divertissements. Celle-ci devait en effet comprendre trois articles, toutefois il n'en reste qu'un seul, consacré à la chasse à l'épervier. C'est dans la deuxième distinction qu'on peut trouver un grand nombre d'informations très précieuses sur la vie quotidienne, entre autres sur la cuisine de l'époque. Mais il ne faut pas le détacher de l'œuvre entière dont il fait partie intégrante.

Le texte même, riche en multiples références, fait croire que son auteur était bien instruit. Les citations et les allusions sont la preuve de la connaissance de nombreux ouvrages religieux et laïques. L'auteur mentionne sa bibliothèque où il garde la Bible, la Légende dorée, la Vie des Pères et d'autres ouvrages utiles écrits en français ${ }^{2}$. Il cite en outre les Pères et les Docteurs, Tite-Live et Macrobe, un manuel d'éducation religieuse et morale de 1279: Somme le Roi du frère Laurent, la traduction française du traité moralisateur de Jacques de Cessoles Liber super ludo scaccorum, Roman de sept sages, le traité pour les femmes mariées de Renaut de

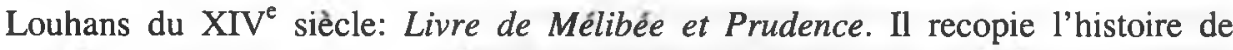
Grisélidis dans la version de Pétrarque traduite par Philippe de Mézières et transcrit intégralement la Voie de Povreté ou de Richesse de Jacques Bruyant de 1342.

Le bonheur n'est pas le thème principal du Mesnagier, il n'est même pas évoqué de façon directe. Le bonheur ou la félicité restent un objectif présupposé des conseils donnés à la jeune épouse. Le but principal de tous les enseignements est l'ordre. Le bonheur est son corollaire. La vision de la félicité esquissée dans le Mesnagier de Paris se concentre autour de l'image de la maison qui à plusieurs reprises est signalée par les termes: maison, mesnagier, mesnaige, mesgnie. Étymologiquement, le mot mesnage désigne à la fois la demeure et ses habitants, la famille et un certain ordre social ${ }^{3}$. Le projet de l'auteur bourgeois est donc de construire à travers l'agencement de l'espace domestique dans sa dimension matérielle et symbolique une vision de la vie digne et honnête.

La maison que l'on peut reconstituer d'après le texte est riche et opulente. Sa structure se montre complexe: l'intérieur de la maison se compose de nombreuses

${ }^{2}$ Voilà ce qu'il dit à la fin de l'article consacrè à l'enseignement catéchistique et moral: «Que tout cela, chère amie, vous suffise en ce qui concerne ce sujet. L'intelligence naturelle dont Dieu vous a dotée, vos bonnes et pieuses dispositions à obẻir à Dieu et à son Église, les prédications et les sermons que vous entendrez en votre paroisse et ailleurs, la Bible, la Lëgende Dorée, l'Apocalypse, la Vie des Pères et quelques autres bons livres en français que je possède et que vous ètes libre de prendre quand vous voudrez, tout cela vous fournira le reste et complètera en profondeur mon enseignement, selon le bon plaisir de Dieu qui veuille vous y conduire et vous y inciter» (I, ii, vv. 1365-1374).

${ }^{3}$ Voir aussi Histoire de la famille, p. 279. 
salles, des chambres, des caves et des greniers. À l'espace domestique s'ajoutent un potager, des écuries, des étables, des poulaillers. Pour mieux comprendre le sens de ces signes, retenons la formule de Philippe Contamine: «Car indépendamment même des dimensions d'une maison, de son mode de construction, de sa situation dans l'espace urbain, de son décor intérieur ou extérieur, de son ameublement, la distribution et la dénomination des pièces dont elle se compose renseignent sur le genre de vie, disons le standing, de son ou de ses occupants. (...) Disposer d'une étable à chevaux ou à mules est le signe qu'on ne se déplace pas à pied dans les rues» ${ }^{4}$. Le nombre de pièces et de dépendances démontre donc la richesse et le bien-être des habitants et contredit la vision stéréotypée de la demeure médiévale primitive et non confortable ${ }^{5}$.

Les préoccupations de l'auteur du Mesnagier se focalisent sur la bonne organisation de l'espace de la maison, il pense tout le temps à son ordre et à sa propreté. Il instruit son épouse qu'il faut «qu'elle ordonne aux chambrières de bien balayer et de tenir impeccable l'entrée de votre maison (à savoir la salle principale et les autres pièces par où les gens passent et où ils s'attardent pour discuter). De même, les tabourets et les différentes housses qui se trouvent sur les sièges doivent être époussetés et secoués. Ensuite les pièces restantes doivent être nettoyées et rangées pour la journée de la même manière, et ainsi chaque jour, comme il sied à notre état» (II, iii, 201-209) ${ }^{6}$. Dans ces gestes, il est possible de reconnaître la volonté - si caractéristique pour la civilisation urbaine - de «civiliser» ou de «domestiquer» l'espace de la maison?

L'image de la maison ne s'épuise pas dans sa structure matérielle, elle renferme aussi une hiérarchie humaine parfaitement organisée. L'épouse y est placée très haut; plus bas, conformément à leur rang se trouvent les domestiques: dame Agnès la béguine, Jean, l'intendant, Robin le berger, Josson le bouvier, Arnoul le vacher, Jeanneton la laitière et Endeline qui s'occupe des volailles.

${ }^{4}$ Histoire de la vie privée, vol. 2 sous la dir. G. Duby, Seuil, Paris 1999, p. 466-467.

5 «Remontant à une très ancienne tradition, d'origine plus ou moins romantique cette fois encore, l'image d'une maison de torchis, à colombages, petite, chétive et mal assurée, s'impose encore, malgré nous, à l'esprit. Cette image correspond bien au schếma général d'une médiocrité des techniques médiévales; toujours solidement ancrée elle évoque les rues obscures, tortueuses, sales, les bâtisses fragiles, irrégulières, sans confort...; ce folklore rejoint les idées toutes faites sur l'insécurité des temps, leur dureté». (J. He e r s, Le Clan familial au Moyen Age, Paris, PUF, 1974, pp. 138-139). Cf. aussi W. R y b c z ń s ki, Dom. Krótka historia idei, rozważania o intymności i prywatności, Gdańsk Warszawa, Marabut - Volumen, 1996, pp. 39-40.

${ }^{6}$ Toutes les citations dans la traduction en français moderne par K. Ueltschi selon l'édition parue dans la collection «Lettres Gothiques», LGF, Paris 1994.

${ }^{7}$ "Á mesure que l'on avance dans les XIII', XIV" siècles, et d'abord en ville, l'outillage ménager se perfectionne, achevant de donner forme à ce qui sera jusque vers 1900 , l'«intérieur" bourgeois, espace parfaitement domestiqué, au prix de sa signification profonde». (P. Z u m t h o r, La Mesure du Monde, Seuil, Paris 1993, p. 86). 
Chaque personne possède une fonction, mais les relations entre les tous ressemblent à une structure organique et solidaire: «Et regardez comment dame Agnès la béguine du côté des femmes, maître Jean l'intendant du côté des hommes distribuent les tâches à vos gens, les faisant travailler partout, l'un en haut, l'autre en bas; l'un aux champs, l'autre en ville; l'un dans les chambres, l'autre à l'étage ou à la cuisine. Ils enverront l'un ici, l'autre là, chacun selon sa place et sa compétence» (II, iii, 407-413).

Les hommes et les objets possèdent donc leur place dans la hiérarchie domestique et c'est ainsi que l'organisation de la maison devient en quelque sorte le reflet de l'harmonie du monde ${ }^{8}$. Il convient de préciser que l'ordre spatial coincide avec l'ordre temporel. À l'exemple du chapitre consacré à l'entretien du jardin qui embrasse tout le cycle annuel depuis la Toussaint jusqu'à la Toussaint on peut voir comment l'ordre de la maison s'inscrit dans l'ordre de la nature (II, ii).

L'avènement même de l'ordre a pu être déjà une source du bonheur, mais c'est l'organisation de l'espace qui rend le bonheur possible. Il est donc temps de montrer ses deux dimensions: celle du bonheur matériel qui combine le plaisir physique avec le confort, et celle du bonheur spirituel qui se manifeste dans le mariage.

Le bonheur qui se réalise dans l'espace du bien-être matériel, se concentre autour du feu, source de la chaleur et de la lumière. Cet aspect matériel et physique rattache le bonheur au sentiment de la sécurité et du rassasiement, aux plaisirs du corps et au confort. L'image de l'homme, assis confortablement près du feu, se régalant de la bonne chère, reste un des fantasmes préférés des rescapés de la grande peste et des troubles de la guerre de Cent Ans. Il est évoqué par les historiens contemporains ainsi que par les poètes d'antan. Johan Huizinga commence ainsi L'Automne du Moyen Age: «Quand le monde était de cinq siècles plus jeune qu'aujourd'hui (...). On jouissait plus avidement de la richesse et des honneurs, car ceux-ci contrastaient plus encore que de nos jours avec la misère environnante. Un tabard fourré, un feu clair, vin et joyeux propos, un bon lit: ces choses offraient encore cette plénitude de bonheur...»". Songeons aussi à François Villon et la ballade Les Contredits Franc Gontier avec le célèbre refrain: «Il n'est tresor que de vivre à son aise». Non sans raison donc la partie culinaire occupe un tiers du Mesnagier et non sans raison, non plus, dans la description de la maison, deux lieux ont un statut privilégié: la cuisine et la chambre ${ }^{10}$. Les plaisirs de la table se conjuguent avec ceux du lit.

${ }^{8}$ Voir Ch. K l a p is c h-Zuber, Les femmes et la famille, in: L'Homme médiéval, sous la direction de: J. Le Goff, Seuil, Paris 1989, p. 339.

${ }^{9}$ Traduit du hollandais par J. Bastin, Payot, Paris 1977, p. 21.

${ }^{10}$ Cette hiérarchisation des pièces se trouve confirmée par les miniatures de l'époque, privilégiant les scènes dans la cuisine, la chambre et la salle de repas, trois lieux principaux du confort médiéval. Cf. D. Alex andre, Home, sweet home: confort et bien-être domestique aux XIV et XV siecles à travers les miniatures in: L'Idée de bonheur au moyen âge, Kümmerle Verlag, Göppingen 1990, p. 31-43. 
Le Mesnagier reprend la conception traditionnelle du mariage. L'époux garde la place centrale dans le foyer et tout et tous s'organisent pour lui servir. Combinant la tradition chrétienne avec le modèle grec et romain, ce pater familias dispose de tous les droits à l'égard de sa famille et de ses domestiques ${ }^{11}$. Son autorité fonde l'ordre et empêche le désordre. Notons que l'auteur mentionne le droit de punir son épouse $(\mathrm{I}, \mathrm{ix}, 1-18)^{12}$.

L'opposition structurale masculin/féminin qui recouvre l'opposition spatiale dehors/dedans est liée à la répartition habituelle des rôles: l'épouse est responsable de garder l'ordre établi par l'homme. Elle est «la clé de son agrément» (I, vi, 1522); c'est à elle de maintenir le bonheur à l'intérieur du foyer, alors que: «aux hommes les occupations et les travaux de l'extérieur. Les maris doivent y pourvoir, aller, venir, courir çà et là, qu'il pleuve, vente, neige ou grêle, tantôt mouillé, tantôt sec, tantôt en sueur ou tremblants de froid, mal nourris, mal logés, mal chaussés, mal couchés. Tout cela ne lui fait rien parce qu'il est réconforté en pensant aux soins que sa femme prendra de lui à son retour, aux caresses, aux joies et aux plaisirs qu'elle lui prodiguera ou lui fera prodiguer en sa présence: le déchausser auprès du bon feu, lui laver les pieds, lui donner des chausses et des souliers propres; et le faire bien manger et bien boire, le servir et l'honorer, et puis le faire coucher entre des draps blancs, avec un bonnet blanc, couvert sous de bonnes fourrures, et le combler de joies, de jeux, de cajoleries amoureuses et d'autres secrets que je passe sous silence. Et le lendemain lui préparer une chemise et des habits nouveaux». (I, vii, 12-27).

«Le confort d'un repas traité à la fois sur les modes symbolique et réaliste est donc l'expression même du bonheur légitime, d'un bonheur chrétien» affirme Danièle Alexandre en citant les paroles d'Ecclésiaste: «Ce qui convient le mieux à l'homme, c'est de manger et de boire, et de trouver le bonheur dans tout le travail qu'il accomplit sous le soleil $(5,17){ }^{13}$. "Ce bonheur domestique justement légitimé par la Bible l'est surtout pour l'homme (... Mais pas pour la femme. En tout cas pas de la même manière (...)». Le témoignage des miniatures atteste le

11 "Les deux textes fondamentaux de la doctrine chrétienne en ce domaine sont le $4^{\mathrm{e}} \mathrm{com}$ mandement du Décalogue - Père et Mère honoreras, afin que tu vives longuement (...), et d'autre part, l'Épître aux Éphésiens $(5,22-6,9)$ où saint Paul a établi l'autorité du père de famille sur sa femme, ses enfants et ses domestiques, et les devoirs d'amour et de correction qu'il a envers eux» (J.-L. Fla ndr i n, Familles, parenté, maison, sexualité dans l'ancienne sociêté, Hachette, Paris 1976, p. 117).

12 "Le droit de battre sa femme lui êtait reconnu par la plupart des anciennes coutumes. Celle du Beauvaisis, au XIIIe siècle, disait: Il est bien à l'homme de battre sa femme, sans mort et sans meshaing [blessure] quant ele desnie son mari. Celle de Bergerac permettait de la battre jusqu'à l'effusion de sang, pourvu que ce füt à bonne intention - bono zelo - pour la corriger. De même celle de Troyes. En 1404, celle de la vallée de Barrèges stipulait: Tout maître et chef de maison peut châtier femme et famille sans que nul ne puisse y mettre obstacle». (Ibidem, p. 122).

${ }^{13}$ V. Home, sweet home..., p. 34. 
contraste entre l'homme siégeant sur son fauteuil et la femme industrieuse à ses pieds $^{14}$.

Un tel bonheur conjugal qui ne peut se réaliser que dans l'espace du foyer est fondé sur le respect et la soumission totale de l'épouse: «il n'existe aucun mari, aussi indigne soit-il, qui ne veuille être obéi et distrait par sa femme» (I, vi, 12881289). Le lecteur n'est donc point étonné de voir combien de place, l'auteur consacre à la question de l'obéissance féminine qui constitue la qualité fondamentale de l'épouse idéale, soutenue par l'autorité biblique et évangélique. C'est à ce titre que sont évoquées les femmes de l'Ancien Testament: Sarah, Rachel et Léa, pour la même raison sont citées les paroles de saint Paul ${ }^{15}$ et multipliés des exempla. Mais la soumission absolue n'est pas suffisante, car l'époux du Mesnagier veut être aimé et veut que son épouse soit amoureuse de lui. Il y revient à plusieurs reprises dans ses consignes: «Pour cette raison, je vous prie d'être très amoureuse et très proche de celui qui sera votre mari) (I, v, 514-515). On pourrait croire que le bonheur conjugal se présente comme une liaison intime de deux personnes réunies exclusivement par l'amour ${ }^{16}$.

Cette félicité intime se traduit sur le plan spatial de la maison par l'harmonie de l'espace concentrique où l'époux se trouve au centre et par l'intermédiaire de son épouse maintient la stabilité de la demeure et de l'entourage. Pour accomplir cet idéal, il n'arrête pas de lui donner des conseils: «Vous devez aimer par-dessus toutes les autres créatures votre mari et être profondément unie à lui; aimez avec modération les meilleurs et les plus proches de vos parents ainsi que ceux de votre mari et accordez-leur votre confiance avec mesure» (I, v, 2-5). Il ajoute dans un autre lieu: «Vous savez bien que votre mari représente votre principale demeure, votre première préoccupation, votre amour et compagnie essentiels» (I, vi, 13201322) et il précise: «quand deux personnes intègres et bonnes sont mariées, toutes les autres attaches affectives sont reléguées au second plan, effacées, oubliées: il n'y a plus qu'eux deux» (I, vi, 1181-1183). Il faut faire attention au fait que l'amour et le souci pour ses propres enfants - que le Mesnagier mentionne dans le contexte

\section{${ }^{14}$ Ibidem, p. 39.}

${ }^{15}$ L'Épître aux Éphésiens 5,22-24 et l'Épître aux Hêbreux 13,17.

${ }^{16}$ Depuis l'Antiquité, les théologiens condamnaient «l'amour de concupiscence» dans le mariage. "Adultère est aussi l'amoureux trop ardent de sa femme», avait écrit saint Jérôme. "Ả l'égard de l'épouse d'autrui, en vérité, tout amour est honteux; à l'égard de la sienne propre, l'amour excessif. L'homme sage doit aimer sa femme avec jugement, non avec passion. Qu'il maîtrise l'emportement de la voluptể et ne se laisse pas emporter avec précipitation à l'accouplement. Rien n'est plus infâme que d'aimer une épouse comme une maîtresse...) (Contre Jovinien - Adversus Iovinianum, I, 49, citế par J.-L. F 1 a n d r i n, Familles..., p. 157).

Selon $\mathrm{H}$. Bresc, c'est l'inégalité de l'âge au mariage qui pose le problème des relations affectives entre époux: «L'amour conjugal semble reposer plutôt sur une sorte de compagnonnage des ếpoux: une amitié adulte, qui n'exclut pas des sentiments de tendresse, mais où l'idée domine qu'un intense amour charnel entre époux équivaut à un adultère» (Histoire de la famille, p. 404). 
des devoirs de l'épouse ${ }^{17}$ - cèdent aussi devant la force de l'amour que les époux doivent avoir l'un à l'égard de l'autre.

Cette exaltation du couple et du bonheur intime fait voir la signification qu'on donne à la chambre conjugale - espace réservé aux époux qui reste à l'abri des regards et des intrus ${ }^{18}$. Dans le Mesnagier le lit se prête aux entretiens et aux confidences les plus secrets: «je me rappelle bien, vous m'avez prié humblement dans notre lit de ne pas vous reprendre brutalement, pour l'amour de Dieu, ni devant des étrangers, ni devant nos gens, mais de le faire au contraire chaque nuit ou au jour le jour dans notre chambre; de vous rappeler alors les fautes ou les naïvetés observées pendant la journée ou auparavant» (Prologue, 9-15). C'est dans l'intimité que l'époux veut voir son épouse lire et écrire des lettres: «Ainsi je vous conseille d'accueillir les lettres personnelles, les lettres d'amour de votre mari avec grande joie et grand respect, de vous isoler pour les lire toute seule, et de lui répondre vous-même de votre main si vous savez écrire, ou alors par l'intermédiaire d'une personne bien discrète. Écrivez-lui de tendres mots d'amour, racontez-lui vos joies et vos passe-temps» (I, iv, 369-375). Dans cet espace secret du foyer, séparé de la sphère publique ${ }^{19}$, se tient la scène dont il est question juste au début du Mesnagier: le mari veut que la jeune épouse danse et chante pour lui et pour ses amis les plus proches mais il lui interdit de le faire devant les invités officiels: «Ainsi, sachez qu'il ne m'est pas désagréable, bien au contraire, que dans l'avenir vous vous occupiez de rosiers, que vous veilliez sur des violettes, que vous en confectionniez des couronnes, et aussi que vous dansiez, que vous chantiez; je souhaite que vous continuiez à le faire devant nos amis et fréquentations: rien de plus naturel et de plus convenable de vous adonner tant que vous êtes une adolescente, à condition de ne pas souhaiter paraître aux fêtes et aux bals de seigneurs trop importants» (Prologue, 28-36).

Mais sous cette image idyllique du bonheur conjugal se cache une angoisse, un pressentiment de la fragilité de ce rêve. Bâti sur l'harmonie idéale entre le dedans et le dehors du foyer, le bonheur reste constamment menacé, précaire. La maison, c'est l'espace parfait qui par définition est opposé au monde et séparé de lui: «La maison c'est l'opposé de l'univers», selon la formule de Paul Zumthor ${ }^{20}$. Le

${ }^{17}$ L'éducation de futurs enfants (I, vi, 1526-1530; II, i, 35-43) et en particulier des filles (Prologue, 63; I, iv, 6-7) semble appartenir au domaine féminin.

${ }^{18}$ La pratique commune était pourtant différente. Dans les maisons des pauvres il y avait une grande promiscuité, les familles couchaient ensemble dans une seule pièce. Quant aux riches, leurs vastes demeures encombrées de domestiques et de visiteurs les empêchaient de vivre dans l'intimité de leurs femmes et de leurs enfants. Voir J.-L. F 1 a n d r i n, Familles..., p. 92.

${ }^{19}$ Contrairement à l'avis de $\mathrm{Ph}$. Ariès: «Toutefois la pièce qui contenait le lit n'en était pas pour autant une chambre à coucher. La chambre demeurait un lieu public», L'enfant et la vie familiale sous l'Ancien Régime, Paris, Seuil, 1973, p. 295. Selon Ariès, on n'a pas de preuve que le besoin d'intimité ait existée avant le XVIII ${ }^{\mathfrak{e}}$ siècle.

${ }^{20}$ Op. cit. p. 82. 
Mesnagier de Paris est basé sur une conception de bonheur sanctionnant une division rigoureuse entre le domaine de l'activité masculine et féminine ${ }^{21}$. Dans les limites de ce partage, l'homme accorde à sa femme une certaine autonomie sans toutefois renoncer à l'exercice du pouvoir sur la totalité de l'espace domestique. Cet univers qu'il a créé du néant est comparé au paradis: "paradis de repos» (I, vii, 181). Puisqu'il ne peut pas y demeurer, il est obligé à confier son bonheur à la femme. Le fonctionnement de la machine compliquée est réglé à l'aide des dispositions, consignes, ordonnances très détaillées qui renferment la femme à l'intêrieur de la maison. Le monde extérieur est l'espace de l'activité masculine: les voyages, les affaires, l'argent et la guerre sont liés à la fatigue et à la peine. Voilà comment l'auteur du Mesnagier instruit sa jeune épouse: «Pour ce qui est des besognes extérieures, reposez-vous sur lui: si c'est un bon mari, il peinera et travaillera même plus que vous ne souhaiteriez» (I, vii, 155-157).

Dans ce monde extérieur, il y a pourtant un ailleurs: «toutes les autres maisons, toutes les autres femmes, toutes les autres attentions, tous les autres foyers» (I, vii, 159-161), exprimant une tentation, un fantasme d'une vie autre, meilleure, des plaisirs hédonistes. Le danger peut venir de l'extérieur: il arrive parfois que les maris, déçus par l'insoumission de leurs épouses «sont mieux obéis ailleurs, ils se consolent avec ces femmes indignes qui savent leur garantir la paix, qui les honorent, qui leur obéissent en toute occasion, qui font toute leur volonté» (I, vi, 1282-1284).

Le projet du bonheur conjugal présente donc des failles. Le rôle de la femme est ambivalent. D'un côté l'auteur articule fortement le rêve d'une épouse - mère nourricière: le bonheur de l'époux est comparé à l'image d'un enfant faible et fragile qui, propre et rassasié, trouve son apaisement à la mamelle d'une femme. L'épouse, tout comme une tendre mère ou nourrice, doit veiller à ce qu'il ait chaud auprès du feu, soit habillé en vêtements et linge propres et raccommodés et qu'il puisse à la fin se reposer blotti, serré contre sa poitrine (I, vii, 41-55). Ce fantasme se trouve parfaitement résumé dans le conseil: «Veillez à ce qu'en hiver il ait un bon feu qui ne fume pas, qu'il soit bien couché entre vos seins sous une bonne couverture» (I, vii, 77-79). Un sens pareil prend la comparaison de l'homme à un cheval ou à un chien dont le propriétaire s'occupe soigneusement, se souciant de leur nourriture et leur pourvoyant une couche propre (I, vii, 162-176). C'est l'épouse qui doit être ce bon maître tandis que le mari se soumet à ses tendres prévenances.

D'autre part, ce qui frappe dans le texte est la fermeté avec laquelle le mari manifeste sa volonté de dominer et de se faire totalement soumettre son épouse. L'amour conjugal est basé sur une obéissance absolue et un dévouement total de la femme à l'égard de son conjoint. Ce désir est exprimé à l'aide de nombreuses

\footnotetext{
${ }^{21}$ Voir Ch. K 1 a p i s c h - Z u b e r, Les femmes..., pp. 338-339.
} 
métaphores animalières dans lesquelles l'époux est à son tour comparé au nourricier et la femme à un animal domestique - chien fidèle à son maître - ou à une bête sauvage dont les instincts sont domptés grâce au dressage: «En observant les animaux domestiques, vous pouvez constater qu'un lévrier, un mâtin, un chiot se tient toujours tout près de celui qui lui donne à manger (...). Même si le chien est loin de son maître, son coeur et son oeil restent toujours avec lui ou sur lui; même si le maître le bat ou lui jette des pierres, cela n'empêche pas le chien de le suivre en remuant la queue» (I, v, 459-466).

Dans la tradition médiévale, le bestiaire de la femme est un bestiaire du mépris et l'on constate une étonnante unanimité quant à l'assimilation de la femme aux animaux malfaisants et nuisibles ${ }^{22}$. Le Mesnagier semble confirmer cette idée du caractère animalier de la nature féminine: «Chère amie, voyez-vous, ce qui est dit des hommes et des femmes vaut également pour les bêtes sauvages, mais encore pour celles qui ont coutume d'emporter et de dévorer leur proie, par exemple les ours, les loups et les lions. On peut les attirer et les apprivoiser en flattant leurs instincts; alors, ils suivent ceux qui les soignent, qui les accompagnent et qui les aiment. Aussi peut-on monter les ours comme des chevaux, ou faire sauter, danser, culbuter les singes ou autres bêtes; elles obéissent à tout ce que leur maître leur demande. À l'aide de cet argument, je vous montre que votre mari vous chérira, vous aimera et vous protégera si vous veillez à tout faire pour le contenter» (I, vi, 1304-1315).

Le mariage est donc un contrat qui peut se retourner en cauchemar si les rôles traditionnellement attribués à l'homme et à la femme ne sont pas respectés. Pourtant l'image idéale ou idéalisée de la femme-épouse a beau cacher une angoisse, un soupçon concernant la nature perverse de la femme, sa perfidie, son insoumission, son indocilité. Cette peur du féminin, renforcée par une longue tradition théologique, s'exprime dans le grand nombre d'interdits et d'avertissements qui pèsent sur le deuxième sexe. La femme ne peut pas ressembler à un homme et l'auteur n'hésite pas à réprouver certaines attitudes: «Il existe des femmes ivrognes, folles ou ignorantes qui ne font pas attention à leur honneur (...); elles marchent les yeux ouverts, la tête effroyablement haute à la manière du lion (... ); elles marchent comme des hommes et se tiennent sans grâce et sans vergogne devant les gens» (I, i, 140-147). Dans le même sens vont les prescriptions concernant la conduite de la femme en dehors de la maison: dans la rue et à l'église. La femme doit marcher en maintenant la tête droite, les paupières baissées, le regard fixant le sol. Elle doit éviter de regarder autour d'elle, elle ne peut pas rire et parler dans la rue. Une fois

${ }^{22}$ «Si nous examinons des listes analogues où c'est la femme qui est comparée aux animaux, le paysage devient tout à coup parfaitement sombre: on pénètre dans une jungle obscure où ne règnent que la griffe, la dent et la perversité: «Femme est lion pour dévorer, / Femme est renard pour décevoir les gens, / Femme est ourse pour recevoir des coups...» (poème le Blasme des fames cite par B. Roy, "La belle e(s)t la bête. Aspects du bestiaire féminin au Moyen Age», Études françaises 10/3, 1974, p. 321). 
arrivée à l'église, elle doit choisir un endroit caché et solitaire, rester là, ne pas changer de place et ne pas se promener (I, ii).

Le bonheur est fragile car l'être humain est imparfait. L'auteur du Mesnagier reste lucide que tout peut basculer en un moment: le paradis domestique risque-t-il de se transformer en une cage ou une prison. L'homme en est, certes, responsable car il peut succomber à la tentation d'un ailleurs. Mais la grande coupable est la femme, à cause de sa nature pervertie, animalière. C'est pourquoi le mari instruit son épouse: «L'on ne rattrape plus facilement un oiseau une fois qu'il s'est sauvé de sa cage; il est plus aisé de l'empêcher de s'envolen» (I, vi, 1298-1300).

Le Mesnagier de Paris présente un modèle du bonheur, montre des conditions dans lesquelles le bonheur peut se réaliser. Il ne s'intéresse pas au bonheur personnel. L'auteur parle rarement de lui-même, il utilise souvent la troisième personne et il se nomme «mari». S'adressant ainsi, au début du livre, à sa jeune épouse, il prend en considération d'autres maris potentiels: «je souhaiterais que vous soyez très instruite en matière de vertu, $d$ 'honneur et de devoirs, et plus encore qu'il ne convient avec moi, pour que vous soyez en mesure de servir, le cas échéant, un autre mari après moi» (Prologue, 59-62). Plus loin il l'avertit: «Sachez (...) belle amie, que si vous avez un autre mari après moi, vous devez très bien vous occuper de sa personne. En effet, lorsqu'une femme a perdu son premier mari et ne vit plus en union conjugale, il est très difficile pour elle de trouver un deuxième qui corresponde à sa condition et à son goût» (I, vii, 3-8).

La conception du bonheur est basée sur une vision du monde en même temps médiévale et bourgeoise. L'idée du caractère stable et immuable des relations entre les sexes et celle de la nature ambivalente des femmes - sont médiévales. Cependant l'exaltation du bonheur conjugal assouplit de façon sensible la rhétorique moralisatrice habituelle. Le besoin du bien-être et du confort matériel, déclaré ouvertement, est lié à la culture urbaine qui se prépare aux fastes de la renaissance. 